\title{
Personality impression formation and sex: The polarization effect
}

\author{
JULIET POPPER SHAFFER \\ University of Kansas, Lawrence, Kansas 66045
}

\begin{abstract}
In a previous experiment, in which subjects rated likability of stimulus persons of both sexes to whom different personality-trait adjectives were ascribed, it was found that ratings were more polarized when stimulus persons were of the opposite sex than when they were of the same sex as the rater; i.e., ascribing positive adjectives resulted in higher likability ratings, while ascribing negative adjectives resulted in lower likability ratings, for opposite-sexed than for like-sexed stimulus persons. This paper reports two further experiments designed to investigate systematically, within an information integration framework, the relationship between the magnitude of this phenomenon and the number and values of the ascribed adjectives. A simple averaging model of information integration does not appear to be adequate to account for the experimental results.
\end{abstract}

In a recent study (Combest, Kasten, \& Shaffer, 1973), male and female college students rated the likability of male or female stimulus persons ("man your age" or "woman your age"), each of whom was described by a single personality-trait adjective. The results indicated greater polarization of likability ratings for opposite-sexed than for like-sexed stimulus persons; i.e., positive traits resulted in higher likability ratings, while negative traits resulted in lower likability ratings, for opposite-sexed than for like-sexed individuals. This phenomenon will be termed the polarization effect. Aside from this overall trend, and contrary to expectation, there was no evidence that any particular traits resulted in different likability ratings when applied to male, as opposed to female, stimulus persons.

The results appeared to be interpretable in terms of a simple version of the stimulus-averaging model of information integration which has been successfully applied in other personality impression formation research (Anderson, 1968b). In that simple version, likability ratings can be predicted by the equation:

$$
\mathrm{R}=\frac{\mathrm{kw} \overline{\mathrm{A}}+(1-\mathrm{w}) \mathbf{I}_{0}}{\mathrm{kw}+(1-\mathrm{w})}
$$

where $R$ is the rating, $\bar{A}$ is the average scale value of the adjectives, $w$ is the weight of each adjective scaled so that the sum of weights in a single-adjective rating problem would be 1 , and $\mathrm{J}_{0}$ is the scale value of an initial impression.

The right side of Equation 1 is a weighted average of the adjective scale values, each equally weighted, and the scale value of the initial impression. Assuming that ratings of likability for both same sex

Ihe author wishes to thank Dorothy Crooks for assistance in conductume the experiments. and opposite sex stimulus persons could be predicted by equations of this form, either of two simple assumptions might account for the obtained result:

1. Differential weight assumption: The weight of the adjectives relative to the initial impression is greater in rating opposite-sexed than same-sexed stimulus persons.

2. Scale value shift assumption: Scale values of the adjectives are more polarized in rating opposite-sexed than same-sexed stimulus persons.

If Assumption 1 were correct, it would follow that the magnitude of the polarization effect should be an increasing function of the extremity of adjectives, i.e., of the absolute difference between their scale values and the neutral value. If Assumption 2 were correct, it would follow that the polarization effect should increase in magnitude with the number of adjectives in the description.

This paper reports the results of two experiments designed primarily to test these assumptions.

In the study reported in Combest et al. (1973), each subject rated 200 stimulus persons, all of the same sex, each of whom was described by one of the 200 starred adjectives recommended for personality impression formation research by Anderson (1968a). Experiment I was designed to investigate more systematically the effect of moderate vs. extreme values of adjectives (relevant to Assumption 1), as well as to determine whether or not the polarization effect occurred when each subject rated stimulus persons of both sexes and also when each rated both sexes on the same adjectives, and whether order of rating had any effect. Experiment II also allowed the comparison of the effects of moderate and extreme adjectives but was carried out primarily to investigate the relationship between the polarization effect and the number of adjectives of approximately equivalent 
Table 1

Means for Different Combinations of SS, SP, and $P N$ in Experiments I and II

\begin{tabular}{lccccc}
\hline \multirow{5}{*}{$\begin{array}{l}\text { Sex of } \\
\text { Subject }\end{array}$} & \multicolumn{2}{c}{ Negative Adjectives } & & \multicolumn{2}{c}{ Positive Adjectives } \\
\cline { 2 - 3 } \cline { 5 - 6 } & Female SP & Male SP & Female SP & Male SP \\
\hline Female & 1.576 & 1.499 & 4.307 & 4.357 \\
Male & 1.607 & 1.760 & 4.324 & 4.251 \\
& \multicolumn{4}{c}{ Experiment I } \\
Female & 1.537 & 1.283 & 4.394 & 4.433 \\
Male & 1.523 & 1.549 & 4.414 & 4.130 \\
\hline
\end{tabular}

*Excluding four-adjective combinations for extreme adjectives.

value attributed to the stimulus person (relevant to Assumption 2).

\section{EXPERIMENT I}

\section{Method}

Materials, procedure, and design. Sixty adjectives were chosen from the set of 200 adjectives used in the previous study in such a way as to provide four ranges of scale values, 15 adjectives in each range, roughly symmetrical in value around 3, the midpoint of the rating scale. Anderson's (1968a) scale values for each range are as follows: extreme positive (4.94-5.29), moderate positive (3.34-4.11), moderate negative (2.09-2.60), and extreme negative (.72-1.04).

Two lists of 40 adjectives each were constructed by randomly choosing 10 out of the 15 words in each range for List $A$, and randomly choosing 5 out of those 10 words to add to the 5 remaining words for List $\mathrm{B}$. Thus, 20 words appeared in both lists, while the other 20 in each list were replaced by words of equivalent value range in the other list.

A single random order of the words in each list was constructed. As in the previous experiment, subjects rated the likability of stimulus persons each of whom was described by a single adjective. Each subject rated likability of a stimulus person of specified sex using one list before rating likability of a stimulus person of the other sex based on adjectives in the other list. Likability was rated by making a slash at any point on a dotted line on which the left end was labeled "very unlikable" and the right end was labeled "very likable." Ratings were recorded in units of $1 / 10$ on a scale from 0 to 6.

The experiment employed a mixed analysis of variance design, with three between-subject variables: (1) sex of subject (SS); (2) list combination (LC): List A with male stimulus person and List $B$ with female stimulus person, or vice versa; and $(3)$ order $(O)$ : male stimulus person first or female stimulus person first; and four within-subject variables: (1) sex of stimulus person (SP), (2) repeated vs. nonrepeated adjectives (RE), (3) positive vs. negative scale value of adjectives (PN), and (4) moderate or extreme scale values (ME); with the mean of the five appropriate adjectives for each subject used as a score on each combination of the latter four variables.

Subjects. The subjects were college students fulfilling a research participation requirement in introductory psychology. The four male subgroups defined by the between-subject variables LC (list combination) and $O$ (order) consisted of from 11 to 13 subjects each, while the four female subgroups consisted of from 6 to 7 subjects each, for a total $n$ of 73 .

\section{Results}

An unweighted means analysis of variance of the data of Experiment I was carried out. The polarization effect, measured by the interaction of sex of subject, sex of stimulus person, and polarity of adjectives, i.e., by the SS by SP by PN interaction, was highly significant, $F(1,65)=8.62, \mathrm{p}<.01$. The magnitude of the effect was not significantly affected by the list combination, the order of presentation of stimulus persons, whether repeated or nonrepeated adjectives are considered, or whether the first stimulus person was being rated or the second in the context of having already rated a stimulus person of the other sex, $F(1,65)=2.12, .56, .91$, and .03 , respectively. As in the previous experiment, there is no evidence for differences in the overall average values of the ratings due to the sex of the rater or to the sex of the stimulus person, $F(1,65)=.39$ and .22 , respectively.

If the model represented by Equation 1 applies, and Assumption 1 holds, the magnitude of the polarization effect should be an increasing function of the extremity of the adjectives. However, the interaction of the effect with extremity of adjectives is not significant, $F(1,65)=.18$; while the magnitude was slightly greater when adjectives were extremely positive than when they were moderately positive, it was smaller when the adjectives were extremely negative than when they were moderately negative.

Means for different combinations of sex of subject, sex of stimulus person, and positive vs. negative adjectives are given in Table 1 .

\section{EXPERIMENT II}

\section{Method}

Materials and procedure. The number of adjectives ascribed before rating likability was varied in Experiment II in a manner similar to that used by Anderson (1967) in his Experiment I. A number of sets of six adjectives each were used in constructing the stimulus series. The stimuli in any such set will be designated $a, b$, $c, d, e$, and $f$.

Practice trials. Four six-adjective sets, two consisting of highly positive and two of highly negative adjectives, were used in constructing the 12 practice items. One single-adjective description, one three-adjective description, and one six-adjective description were constructed from each set by using the combinations e, bcd, and abcdef, making a total of 12 practice trials. Combinations derived from one positive set and one negative set were each ascribed to a "woman your age," and those from the other positive and negative sets to a "man your age," with the particular sex-set combinations counterbalanced. The four practice sets were: imaginative, courteous, enthusiastic, unselfish, original, responsible; helpful, pleasant, appreciative, broad-minded, patient, cheerful; superficial, unpleasant, jealous, boring, untruthful, impolite; and unreliable, distrustful, abusive, ill-mannered, crude, quarrelsome.

Extreme-value adjectives. Four six-adjective sets, each containing adjectives of approximately equal value, were used in constructing a series of extreme-value descriptions. Twelve descriptions, each based on one, three, four, or six adjectives, were constructed from each set by using the combinations $a, b, c, d, e, f$, abc, def, abcd, abef, cdef, and abcdef, making a total of 48 such descriptions. Descriptions derived from one positive and one negative set were each ascribed to a "woman your age," and those based on the other positive and negative set to a "man your age," as in the case of the practice items.

The four sets were the same as those used in Experiment $I$ of Anderson (1967), i.e., thoughtful, wise, considerate, good-natured, 
reliable, mature; warm, earnest, kind, friendly, happy, interesting; spiteful, annoying, conceited, narrow-minded, disrespectful, greedy; and rude, thoughtless, vulgar, heartless, selfish, loud-mouthed. Descriptions based on the first positive and first negative sets above constituted the extreme descriptions in List A, while those based on the second positive set and the second negative set constituted the extreme descriptions in List B.

Moderate-value adjectives. Four six-adjective sets were used in constructing a series of moderate-value descriptions. Six descriptions, each based on one, three, or six adjectives, were constructed from each set by using the combinations $d, e, f, a b c$, def, and abcdef, making a total of 24 such stimulus combinations. Combinations based on one positive and one negative set were each ascribed to a "woman your age," while those derived from the other positive and negative sets were each ascribed to a "man your age." The four sets were: orderly, excited, persistent, persuasive, cautious, idealistic; sentimental, serious, bold, proud, self-assured, thrifty; critical, sarcastic, forgetful, lonely, timid, gullible; and self-conscious, indecisive, materialistic, rebellious, absent-minded, unemotional. Descriptions based on the first positive and first negative set above constituted the moderate descriptions in List A, while those based on the second positive and second negative set constituted the moderate descriptions in List B.

In the rating booklets, each combination of adjectives appeared on a different page, and the pages were independently randomized for each subject, with the 12 practice stimuli in random order, followed by the 72 other descriptions (48 extreme, 24 moderate) in random order. Ratings were made as in Experiment $I$.

Subjects. The subjects were students in introductory psychology, some of whom were fulfilling a research requirement and some of whom were paid for participation. There were 24 subjects per experimental subgroup, or a total of 96 .

\section{Results}

The major results of Experiment II are illustrated in Figure 1. Since only three of the adjectives in each six-adjective set were presented singly for the moderate adjectives, the curves for those adjectives are in two segments-the first giving the mean of the three single-adjective presentations and the mean when rating those same three adjectives presented jointly, and the second segment giving the mean of the two triple-adjective presentations and the mean of the presentation of all six adjectives simultaneously. Thus, within each segment, the same adjectives have been used. By inspection, it did not appear that the conclusions with respect to polarization would be affected by this lack of balance in the design for moderate items, so that analyses to be reported used the three-adjective ratings based upon the two three-adjective combinations, i.e., the values from the second segment of the curve. As a check, analyses of variance were carried out separately on the two segments of the curve, but these did not yield any results contrary to those based on the combined analyses, and they will not be considered further.

An overall analysis of variance, combining moderate and extreme adjectives, was carried out by ignoring the four-adjective combinations for extreme adjectives, and therefore using the Values 1,3 , and 6 for the number of adjectives being rated. The design is mixed, with two between-subject factors, LC and SS, and four within-subject factors, ME, SP, PN, and number of adjectives (NA). The polarization effect, SS by SP by PN, is highly significant, $F(1,92)=19.18$,

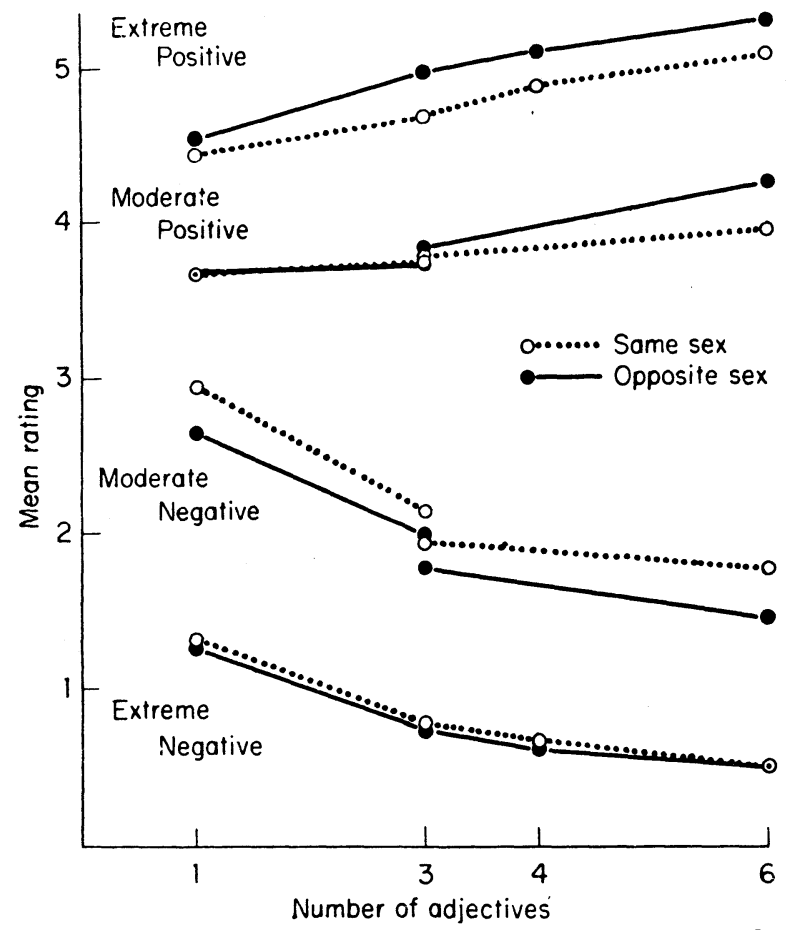

Figure 1. Mean ratings of like- and opposite-sexed stimulus persons as a function of number and value of adjectives in Experiment II.

$\mathrm{p}<.001$. It is significant for negative adjectives alone, $\mathrm{F}(1,92)=10.15, \mathrm{p}<.01$, and for positive adjectives alone, $F(1,92)=8.00, p<.01$. As in Experiment I, there is no overall relationship between the magnitude of the polarization effect and extremity of adjective scale values, $F(1,92)=1.24$; for positive adjectives, there is a nonsignificant positive relationship between magnitude and extremity, $F(1,92)=.95$; while for negative adjectives, there is a significant negative relationship, i.e., moderate adjectives resulted in a larger polarization effect than did extreme adjectives, $F(1,92)=7.67, p<.01$.

If the model and Assumption 2 holds, the magnitude of the polarization effect should increase with number of adjectives. However, the interaction between the polarization effect and number of adjectives is not significant, $F(2,184)=1.26$, and inspection of Figure 1 does not suggest any subtle relationship between the effect and the number of adjectives to which the overall $F$ test would not be sensitive.

While in the previous experiment, and in Experiment I, there was no significant difference between the average ratings of female and male stimulus persons over all adjectives and subjects, in Experiment II the difference is highly significant, $F(1,92)=9.88, p<.01$, with women as stimulus persons rated more positively overall than men. Since, in both other experiments, the difference, although insignificant, is in the opposite direction, further investigation would be desirable before attempting to interpret this effect. 
The means for different combinations of sex of subject, sex of stimulus person, and positive vs. negative adjectives over all ratings, except for the four-adjective ratings for extreme adjectives (since there were no corresponding ratings for moderate adjectives) are given in Table 1.

\section{Summary and Discussion}

The results of the three experiments taken together (the two reported here, and the previous one discussed) provide strong support for the existence of the polarization effect: subjects rate stimulus persons of the opposite sex in a more polarized fashion than stimulus persons of the same sex. Analysis of the previous experiment had suggested the possibility of a positive relation between magnitude of the effect and extremity of scale value, which would have supported the simple stimulus averaging model with Assumption 1 as a possible explanation of the results. The failure to find support for such a polarization effect-extremity relationship in Experiments I and II, and the evidence for a reverse relationship in the case of negative adjectives, make this interpretation of the polarization effect untenable. Similarly, the results of Experiment II provided no support for the prediction derived from Assumption 2 that the polarization effect should be an increasing function of the number of adjectives.

It might be possible to account for the details of the relationship between the polarization effect and the polarity, extremity, and number of adjectives in terms of the model (Equation 1) by assuming a difference in both weights and scale values when rating individuals of the opposite sex as opposed to individuals of the same sex, but this would not provide a very parsimonious or satisfying explanation. Alternatively, the obtained relationships might be explicable in terms of a more complex information integration model, e.g., one which allowed different weights for different adjectives.

In conclusion, the results of the experimental work indicate the existence of an intriguing difference in likability ratings for the same and the opposite sex. The attempt to account for those results in detail within the framework of a simple information integration model was unsuccessful.

\section{REFERENCES}

Anderson. N. H. Averaging model analysis of set size effect in impression formation. Journal of Experimental Psychology, 1967, 75, 158-165.

ANDERSON, N. H. Likableness ratings of 555 personality-trait words. Journal of Personality and Social Psychology, 1968. 9. 272-279. (a)

ANDERSON, N. H. A simple model for information integration. In R. P. Abelson, E. Aronson, W. J. McGuire, T. M. Newcomb, M. J. Rosenberg, \& P. H. Tannenbaum (Eds.), Theories of cognitive consistency: $A$ sourcebook. Chicago: Rand McNally, 1968. (b)

Combest, W., Kasten, K., \& Shaffer, J. P. The relationship between personality impression formation and sex: An application of information integration theory. Bulletin of the Psychonomic Society, 1973, 1. 2-4.

(Received for publication August 8, 1975.) 